\title{
Produção de estolhos de cultivares de morangueiro em função da condutividade elétrica da solução nutritiva
}

\author{
Odair J Schmitt; Jerônimo L Andriolo; Erani Schultz; Maíne A Lerner; Jéssica M Souza; Miriane Dal \\ Picio \\ Universidade Federal de Santa Maria, Depto. Fitotecnia, (UFSM), Santa Maria-RS, Brasil; odairschmitt@yahoo.com.br (autor para \\ correspondência); jeronimoandriolo@gmail.com; erani schultz@hotmail.com; mainelerner@yahoo.com.br; jessica_maronez@hotmail. \\ com; mirianedalpicio@yahoo.com.br
}

\section{RESUMO}

As características da solução nutritiva empregada em sistemas de cultivo sem solo com uso de substratos é um dos fatores que influenciam a emissão de estolhos. A condutividade elétrica (CE) da solução nutritiva está relacionada com concentração de nutrientes na solução nutritiva, mas ainda pouco relatada na literatura para produção de mudas de morango no Brasil. Assim, o objetivo do trabalho foi determinar o efeito da condutividade elétrica da solução nutritiva no crescimento de plantas matrizes de morangueiro e na produção de estolhos para produção de mudas com torrão. O delineamento experimental foi inteiramente casualizado, esquema fatorial $(5 \times 2)$, sendo as CEs de 0,$4 ; 0,8 ; 1,2 ; 1,6$ e $2,0 \mathrm{dS} / \mathrm{m}$ e as cultivares de morangueiro a Camino Real e Oso Grande, com 20 repetições de uma planta matriz. O plantio foi feito em vasos com areia, sendo as plantas fertirrigadas com solução nutritiva nas cinco condutividades elétricas. Os estolhos foram coletados semanalmente e contabilizados, sendo descartados aqueles necrosados. Quinzenalmente, foram coletadas amostras de 15 estolhos de cada tratamento, nas quais foi determinado o número de folhas, diâmetro de coroa e massa seca. Em fevereiro, uma amostra de 45 estolhos coletados em cada tratamento foi enraizada em bandejas de poliestireno contendo $40 \mathrm{~cm}^{3}$ de substrato orgânico para determinação da taxa de formação de mudas, do diâmetro de coroa, do número de folhas expandidas e da massa seca da parte aérea e das raízes. Ao final do experimento, foram coletadas, também, plantas matrizes para determinação da área foliar e da massa seca de raízes, coroas, pecíolos e folhas. Concluiu-se que o aumento da condutividade elétrica reduziu o crescimento das plantas matrizes e a produção de estolhos e de mudas com torrão foram maiores com valores de CE na faixa entre 0,8 e $1,1 \mathrm{dS} / \mathrm{m}$.

Palavras-chave: Fragaria $x$ ananassa, propagação, mudas, fertirrigação, solução nutritiva.

\begin{abstract}
Yield of strawberry runner tips under different electrical conductivity of the nutrient solution

The characteristics of the nutrient solution used in soilless culture systems with the use of substrates is one of the factors which influence the emission of runner tips. The electrical conductivity (EC) of the nutrient solution is related to the concentration of nutrients, but still few reported in the literature for production of strawberry plug transplants in Brazil. The objective of this research was to determine the effects of the nutrient solution electrical conductivity on growth of strawberry stock plants and on production of runner tips for plug transplants. The completely randomized experimental design was used in a $(5 \times 2)$ factorial scheme. Five concentrations of the nutrient solution measured by means of its EC of $0.4 ; 0.8 ; 1.2 ; 1.6$ and 2.0 $\mathrm{dS} / \mathrm{m}$ and strawberry cultivars Camino Real and Oso Grande were compared, with 20 replications of one stock plant. Planting was carried out in pots in October and plants were fertigated with the five concentrations. Runner tips were collected weekly and counted, necrotic ones being discarded. A sample of 15 runner tips from plants of each treatment was collected at two-week intervals to determine the number of leaves, crown diameter and dry mass. In February, a sample of 45 runner tips from each treatment was rooted in polystyrene trays with $40 \mathrm{~cm}^{3}$ of organic substrate to determine the transplant survival rate, crown diameter, number of expanded leaves, shoot and root dry mass of transplants. At the end of the experiment, leaf area and dry mass of roots, crown, petioles and leaves were determined. We concluded that increasing the concentration of the nutrient solution reduces the growth of strawberry stock plants and the production of runner tips and transplants is higher in the EC range between 0.8 and $1.1 \mathrm{dS} / \mathrm{m}$.
\end{abstract}

Keywords: Fragaria $x$ ananassa, propagation, seedlings, fertirrigation, nutritive solution.

(Recebido para publicação em 23 de janeiro de 2015; aceito em 21 de janeiro de 2016) (Received on January 23, 2015; accepted on January 21, 2016)

$\mathrm{Si}$ istemas de cultivo sem solo são recomendados e amplamente utilizados na produção de mudas de morangueiro com torrão na Europa e Estados Unidos (Bish et al., 2001; Durner et al., 2002; Armeflhor, 2006). Para a produção de mudas com torrão em sistemas de cultivo sem solo é necessário um grande número de estruturas vegetativas jovens (estolhos) viáveis (Schmitt et al., 2012), sendo cada estolho formador de uma única muda. Isso é diferente dos sistemas de produção de mudas de raízes nuas, em que um estolho emitido e enraizado origina várias mudas.

$\mathrm{Na}$ região Sul do Brasil, produção de mudas de morangueiro é alternativa à importação de mudas do Chile e Argentina, as quais apresentam custo elevado, sofrem danos no transporte, com qualidade fisiológica e sanitária 
duvidosa e principalmente, atrasos na entrega aos produtores (Janisch et al., 2012; Andriolo et al., 2014). O cultivo sem solo e a produção de mudas com torrão permitem formação de mudas com alta qualidade, baixa infestação por patógenos e plantio na época adequada, proporcionando, assim, aumento na produção de frutos na entressafra (Giménez et al., 2008; Janisch et al., 2012) com maior lucro para os produtores.

A produção de estolhos de morangueiro em sistemas de cultivo sem solo com uso de substratos está relacionada com a cultivar, as condições ambientais, a composição e características da solução nutritiva empregada (Durner et al., 2002; Janisch et al., 2012). A solução nutritiva deverá fornecer os nutrientes de forma equilibrada para a planta matriz, possibilitando a máxima emissão de estolhos, sem comprometer sua qualidade fisiológica e viabilidade para o enraizamento.

O crescimento das plantas é influenciado pela condutividade elétrica (CE), que é uma medida indireta da concentração salina da solução nutritiva e está relacionada com a concentração de nutrientes da solução nutritiva (Andriolo et al., 2009; Sonneveld \& Voogt, 2009). O morangueiro é considerado uma planta sensível a elevadas $\mathrm{CE}$, não tolerando valores superiores a 1,4 dS/m (Martinez Barroso \& Alvarez, 1997). No cultivo do morangueiro sem solo para produção de frutos tem sido recomendada a CE de 0,9 $\mathrm{dS} / \mathrm{m}$ quando o sistema utiliza substratos (Andriolo et al., 2009) e 1,2 a 1,5 dS/m no sistema NFT (Portela et al., 2012).

Nos Estados Unidos, as condutividades elétricas recomendadas para a produção de estolhos de morangueiro são bastante distintas, variando de 0,7 $\mathrm{dS} / \mathrm{m}$ (Bisch et al., 2001) a 1,6 dS/m no cultivo de plantas matrizes para a produção de estolhos em cultivo sem solo (Paranjpe et al., 2003). Entretanto, resultados ainda são escassos na literatura, especialmente brasileira, relativas à condutividade elétrica e sua respectiva concentração da solução nutritiva que favoreça a produção e a qualidade de estolhos em sistemas de cultivo sem solo de morangueiro.

O objetivo do trabalho foi determinar o efeito da condutividade elétrica da solução nutritiva no crescimento das plantas matrizes de morangueiro, na produção e na qualidade dos estolhos para obtenção de mudas com torrão.

\section{MATERIAL E MÉTODOS}

O experimento foi conduzido no interior de um abrigo de $200 \mathrm{~m}^{2}$ tipo guarda-chuva, com $65 \%$ de superfície de ventilação, coberto com polietileno aditivado anti-UV de $200 \mu \mathrm{m}$ de espessura, localizado no Departamento de Fitotecnia, da Universidade Federal de Santa Maria.

Durante o período experimental (13 de outubro de 2011 a 10 de abril de 2012), a temperatura média do ar e a radiação solar global acumulada foram coletadas, diariamente, por uma estação meteorológica automática distante $300 \mathrm{~m}$ da área experimental. Os valores médios obtidos para essas variáveis foram: $19,03^{\circ} \mathrm{C}$ e $558,6 \mathrm{MJ} /$ $\mathrm{m}^{2}$ (outubro), $22,11^{\circ} \mathrm{C}$ e $718,9 \mathrm{MJ} /$ $\mathrm{m}^{2}$ (novembro), $23,03^{\circ} \mathrm{C}$ e $754,5 \mathrm{MJ} /$ $\mathrm{m}^{2}$ (dezembro), $25,28^{\circ} \mathrm{C}$ e $775,8 \mathrm{MJ} /$ $\mathrm{m}^{2}$ (janeiro), $25,88^{\circ} \mathrm{C}$ e $564,8 \mathrm{MJ} / \mathrm{m}^{2}$ (fevereiro), $22,58^{\circ} \mathrm{C}$ e $621,9 \mathrm{MJ} / \mathrm{m}^{2}$ (março), $21,43^{\circ} \mathrm{C}$ e $159,8 \mathrm{MJ} / \mathrm{m}^{2}$ (até o dia 10 de abril).

As plantas foram cultivadas em vasos de polietileno $\left(2,5 \mathrm{~cm}^{3}\right)$ preenchidos com areia (1 e $3 \mathrm{~mm}$ ) e capacidade máxima de retenção de água de $0,238 \mathrm{~L} / \mathrm{dm}^{3}$. Os vasos foram dispostos sobre bancadas a $80 \mathrm{~cm}$ de altura do piso, com 1,10 $\mathrm{m}$ de largura e $4 \mathrm{~m}$ de comprimento, distribuídos em quatro fileiras, na distância de $30 \mathrm{~cm}$ entre vasos na fileira e $27 \mathrm{~cm}$ entre fileiras, correspondente à densidade de 12 vasos por metro quadrado de bancada. Antes do plantio, os vasos foram cobertos individualmente, utilizando filme dupla-face de $150 \mu \mathrm{m}$ de espessura para reduzir o consumo de água e evitar o aumento da condutividade elétrica (CE) do substrato (Farina et al., 2003) e o enraizamento dos estolhos, anteriormente à coleta. As bancadas foram constituídas por telhas de fibrocimento revestidas com lona de polipropileno, conforme descrito por Godoi et al. (2009).

Foi utilizado o delineamento inteiramente casualizado, em esquema fatorial
$5 \times 2$, sendo cinco condutividades elétricas da solução nutritiva $(0,4 ; 0,8 ; 1,2$; 1,6 e 2,0 dS/m) e duas cultivares de morangueiro (Camino Real e Oso Grande), com 20 repetições de uma planta matriz. Essas cultivares foram escolhidas por apresentarem vigor vegetativo distinto, conforme descrito por Shaw \& Larson (2002) e Voth \& Bringhurst (1989), respectivamente.

A composição da solução nutritiva de referência utilizada foi descrita por Hennion \& Veschambre (1997), ajustada para a concentração em mmol/L: 7,86 de $\mathrm{NO}_{3}^{-} ; 1,86$ de $\mathrm{NH}_{4}^{+} ; 4$ de $\mathrm{H}_{2} \mathrm{PO}_{4}^{-} ; 6$ de $\mathrm{K}^{+} ; 2,0$ de $\mathrm{Ca}^{+2} ; 1$ de $\mathrm{Mg}^{+2}$ e 1 de $\mathrm{SO}_{4}^{-2}$. As concentrações dos micronutrientes foram, em mg/L: 0,03 de Mo; 0,26 de B; 0,06 de $\mathrm{Cu}$; 0,50 de Mn; 0,22 de Zn, fornecidos por meio de uma solução estoque. A concentração do ferro foi 1,0 mg/L, fornecido separadamente na forma quelatizada. Essa solução nutritiva apresentou $\mathrm{CE}$ igual a $1,5 \mathrm{dS} / \mathrm{m}$ e $\mathrm{pH}$ de 5,9. As fontes de macronutrientes foram os fertilizantes nitrato de potássio, fosfato monopotássico, nitrato de cálcio-Calcinit $^{\circledR}$, sulfato de magnésio e nitrato de amônio. As condutividades elétricas testadas foram obtidas por meio da diluição ou concentração proporcional dos componentes da solução nutritiva de referência, a partir de fatores de ajuste, obtidos pela divisão de cada valor de CE desejado $(0,4 ; 0,8 ; 1,2$; 1,6 e 2,0 dS/m) pela CE da solução de referência $(1,5 \mathrm{dS} / \mathrm{m})$ respectivamente, multiplicando-os pela concentração de cada molécula catiônica e aniônica da solução referência.

$\mathrm{A} \mathrm{CE}$ e o $\mathrm{pH}$ foram medidos diariamente. Sempre que o valor medido da CE apresentou um desvio de 5\% em relação à maior $\mathrm{CE}$ testada $(2,0 \mathrm{dS} / \mathrm{m})$, foram feitas correções adicionando água ou alíquotas de nova solução nutritiva nas concentrações de referência. $\mathrm{O}$ pH foi mantido entre os limites de 5,5 e 6,5, mediante adição de $\mathrm{NaOH}$ para aumentar e de $\mathrm{H}_{2} \mathrm{SO}_{4}$ para reduzir, nas concentrações 1 e $0,5 \mathrm{M}$, respectivamente.

As mudas matrizes de ambas as cultivares, foram obtidas a partir de plantas matrizes micropropagadas conforme recomendações de Bish et al. (2001) e Giménez et al. (2008), adquiridas do Laboratório de Micropropagação de 
Plantas da Universidade de Passo Fundo, as quais foram retiradas dos frascos em 16 de setembro de 2011 e plantadas em bandejas de poliestireno com 128 células preenchidas com o substrato orgânico MECPLANT HF ${ }^{\circledR}$. As bandejas foram mantidas em câmara úmida, localizada no interior do abrigo, durante 28 dias para aclimatização. Após este período, foram transplantadas para os vasos na fase de uma folha definitiva.

A água e os nutrientes foram fornecidos por fertirrigação, empregando fita gotejadora, com um gotejador por vaso. A solução nutritiva foi preparada em caixas de polipropileno de $500 \mathrm{~L}$ e fornecida às plantas da bancada por meio de uma motobomba submersa, controlada por um programador horário. Após o plantio, realizou-se uma irrigação diária de 15 minutos, usando somente água por uma semana. Entre os dias 20 de outubro e 28 de novembro, foi realizada uma fertirrigação diária e, posteriormente, duas a três fertirrigações de 15 minutos. $\mathrm{O}$ volume e a frequência diária das fertirrigações foram determinados levando em conta a estimativa da evapotranspiração, calculada com base em Reisser Júnior et al. (2009), com um coeficiente de drenagem de $30 \%$. Todas as plantas da área experimental foram fertirrigadas concomitantemente. O volume de solução nutritiva drenado retornou à caixa de estocagem da solução nutritiva e foi reutilizado, em sistema fechado. O volume de água e quantidade de sais fertilizantes consumidos durante o experimento foram registrados.

Durante o período de emissão de estolhos, que ocorreu entre 11 de novembro de 2011 a 02 de março de 2012, foram coletados semanalmente os estolhos emitidos que apresentaram uma folha definitiva e/ou primórdios radiculares visíveis e os estolhos necrosados, sendo estes contabilizados e descartados.

Dos estolhos viáveis, quinzenalmente foi retirada uma amostra de 15 unidades, nas quais foi contado o número de folhas e medido o diâmetro da coroa. Em seguida, foram separadas em limbo, pecíolo e coroa e posteriormente secas em estufa de circulação forçada de ar à temperatura de $60^{\circ} \mathrm{C}$ até massa constante entre duas pesagens conse- cutivas, para a determinação da massa seca (MS). Ao final do período de coleta de estolhos foi determinado, por planta matriz, o número total de estolhos emitidos, necrosados e viáveis.

No dia 29 de fevereiro de 2012, no período de produção de mudas comerciais, foram coletados 45 estolhos de cada tratamento para avaliação da qualidade das mudas originadas dos estolhos provenientes das plantas de cada tratamento. Imediatamente após a coleta, os estolhos foram enraizados em bandejas de poliestireno, contendo $40 \mathrm{~cm}^{3}$ de substrato orgânico (MECPLANT HF ${ }^{\circledR}$ ), os quais foram mantidos em câmara úmida por 10 dias e, posteriormente, transferidos para bancadas (descritas por Godoi et al. (2009), localizadas no interior do abrigo de polietileno, irrigados diariamente no período diurno por microaspersão durante 1 minuto, a cada hora, durante 30 dias, para enraizamento.

Ao final desse período, calculou-se a taxa de formação de mudas com torrão, sendo assim consideradas aquelas que, ao serem removidas do alvéolo da bandeja, conservaram o torrão de substrato aderido nas raízes. Para a determinação do diâmetro de coroa, do número de folhas expandidas, da massa seca de parte aérea e de raízes das mudas formadas, foram coletadas aleatoriamente 15 mudas de cada tratamento, resultando em cinco repetições, compostas por três mudas.

Ao final do experimento, foram coletadas quatro plantas de cada cultivar em cada um dos tratamentos, totalizando 40 plantas matrizes, as quais foram separadas em limbo, pecíolo, coroa e raiz, lavadas e posteriormente secas em estufa de circulação forçada de ar para a determinação da MS dos diferentes órgãos da planta.

A área foliar específica (AFE) e o índice de área foliar (IAF) foram determinados a partir da relação entre a superfície e a massa seca de 30 discos com $5,02 \times 10^{-5} \mathrm{~m}^{2}$ de diâmetro, extraídos em cada planta. O IAF foi estimado pela relação entre a AFE e MS de folhas.

Os dados foram submetidos à análise da variância. As diferenças entre as médias dos níveis do fator cultivar (qualitativo) foram comparadas pelo teste de Tukey, ao nível de 5\% de probabilidade de erro, e os níveis do fator $\mathrm{CE}$ (quantitativo), por regressão, usando o programa estatístico SISVAR/UFLA versão 4.2 (Ferreira, 2003) e a planilha eletrônica Excel (Microsoft Office). A significância dos $\mathrm{R}^{2}$ das equações de regressão foi testada por meio do teste F, utilizando o software estatístico SPSS 13.0 (SPSS, 2004). As equações foram interpretadas por meio de sua derivação para a obtenção dos pontos de máxima.

\section{RESULTADOS E DISCUSSÃO}

Para o número de estolhos emitidos pelas plantas matrizes das cultivares Oso Grande e Camino Real, observou-se interação significativa entre cultivares e concentrações da solução nutritiva indicadas pela condutividade elétrica (CE) (Figura 1A). A cultivar Oso Grande apresentou número médio de 63,1 estolhos emitidos por planta matriz ao final do experimento, diferindo $(\mathrm{p}<0,05) \mathrm{da}$ Camino Real $(48,9)$. A máxima emissão de estolhos estimada de ambas cultivares foi obtida nas condutividades de 1,0 e $0,8 \mathrm{dS} / \mathrm{m}$ com 73,5 e 54,4 estolhos por planta matriz, respectivamente.

Quanto ao número de estolhos necrosados (Figura 1B), Oso Grande apresentou aumento linear de estolhos necrosados com o aumento da condutividade atingindo 5,2 estolhos necrosados por planta matriz na $\mathrm{CE}$ de $2,0 \mathrm{dS} / \mathrm{m}$, o que correspondeu a $13,7 \%$ dos estolhos emitidos. Camino Real mostrou-se menos sensível ao aumento nas CEs entre 0,4 e 1,2 dS/m, no entanto apresentou um aumento de $14,6 \%$ de estolhos necrosados na condutividade de 2,0 dS/m comparado a CE de 1,2 dS/m.

As plantas matrizes da Oso Grande apresentaram maior número de estolhos viáveis para produção de mudas com torrão na $\mathrm{CE}$ de $0,9 \mathrm{dS} / \mathrm{m}$, totalizando 71,5 estolhos por planta matriz (Figura $1 \mathrm{C}$ ). Camino Real atingiu a maior produção de estolhos viáveis na $\mathrm{CE}$ de $0,8 \mathrm{dS} / \mathrm{m}$ com 54 estolhos por planta matriz. As diferenças entre as cultivares pode ser atribuída à diferença no vigor vegetativo, sendo maior na cultivar Oso Grande, conforme descrito por Shaw \& Larson (2002) e Voth \& Bringhurst 


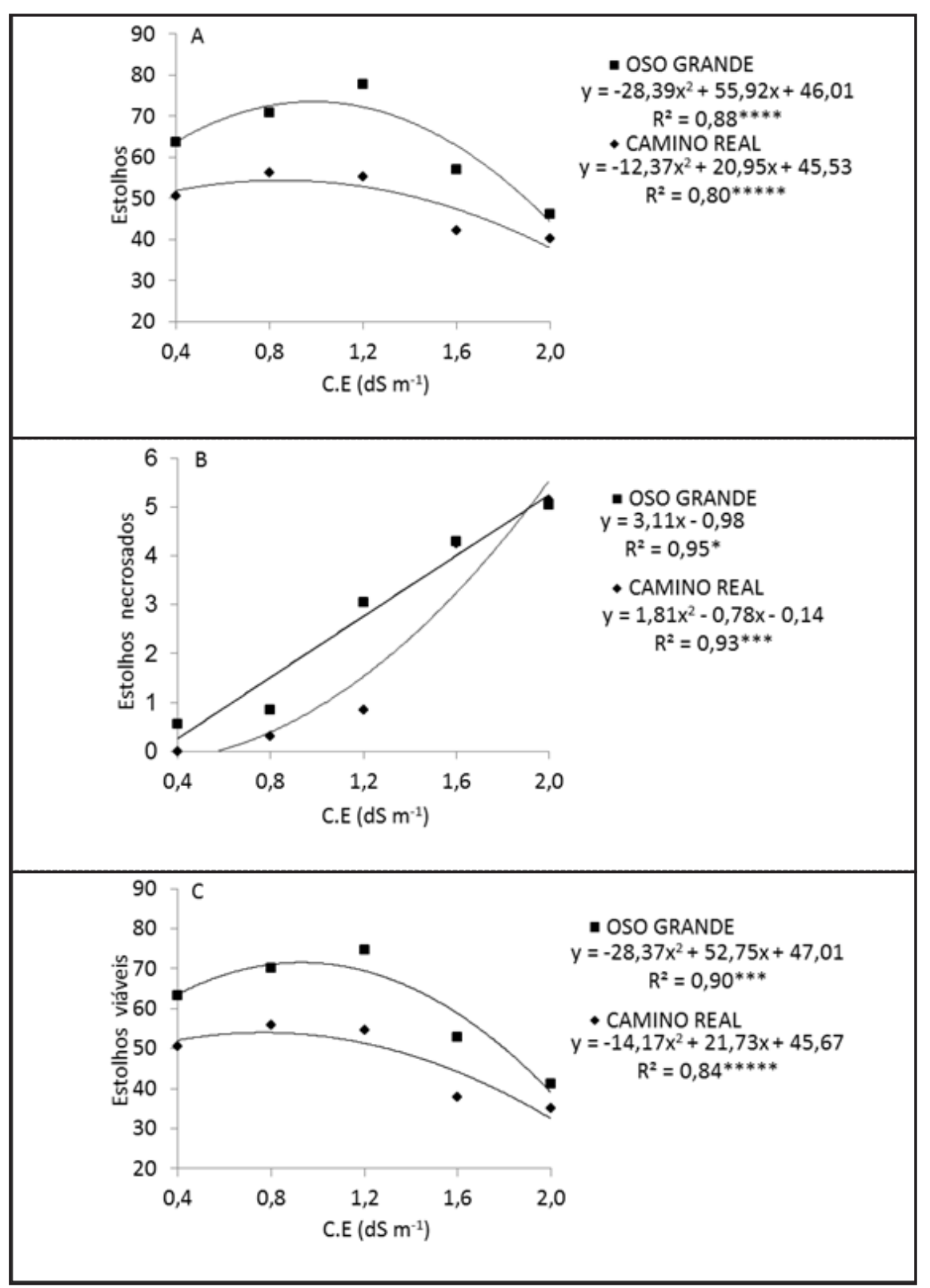

Figura 1. Número de estolhos produzidos $(\mathrm{A})$; necrosados $(\mathrm{B})$ e viáveis $(\mathrm{C})$ de plantas matrizes de morangueiro das cultivares Oso Grande e Camino Real cultivadas sob cinco concentrações da solução nutritiva $(0,4 ; 0,8 ; 1,2 ; 1,6$ e $2,0 \mathrm{dS} / \mathrm{m}) . * * * *, * * * * \mathrm{e} * * * * *=\mathrm{p} \leq 0,01$, $\mathrm{p} \leq 0,10, \mathrm{p} \leq 0,15$ e $\mathrm{p} \leq 0,20$, respectivamente number of runner tips produced (A), necrotic (B) and viables (C) from Oso Grande and Camino Real strawberry stock plants grown under five concentrations of the nutrient solution $(0.4 ; 0.8 ; 1.2 ; 1.6$; and $2.0 \mathrm{dS} / \mathrm{m}) . *$, ***, **** and $* * * * *=p \leq 0.01, p \leq 0.10, p \leq 0.15$ and $p \leq 0.20$, respectively $\}$. Santa Maria, UFSM, 2012.

(1989). Não houve diferença para diâmetro de coroa e massa seca dos estolhos coletados ao longo do período experimental, apresentando médias de $5 \mathrm{~mm}$ e $0,3 \mathrm{~g}$ por estolho.

Embora não tenham ocorrido diferenças entre cultivares nas características avaliadas relativas às mudas formadas com os estolhos coletados, houve efeito das condutividades elétricas da solução nutritiva (Figura 2). O máximo aérea e raízes, o índice de área foliar (IAF) e a área foliar específica (AFE) das plantas matrizes não diferiram entre as cultivares e entre as CEs (Figura 3). Em ambas as características avaliadas houve decréscimo linear em função do aumento da CE. Comparando a CE $0,4 \mathrm{com}$ a $2,0 \mathrm{dS} / \mathrm{m}$, foram verificadas reduções de 65,8 e $55 \%$ (Figura $3 \mathrm{~A}$ ), $72 \%$ (Figura 3B), e 25\% (Figura 3C), respectivamente.

A emissão de estolhos da cultivar Camino Real do presente trabalho (Figura 1A) diferiu de Janisch et al. (2012) obtido no mesmo local. Esses autores mostram redução de 54 (Janisch et al., 2012) para 40 estolhos por planta matriz com 2,0 dS/m. Comparando os dados obtidos no presente trabalho aos de Janisch et al. (2012), as variações dos tratamentos desses autores foram somente nas doses de nitrogênio $(\mathrm{N})$, as quais não apresentaram diferenças quanto à produção de estolhos. No presente trabalho, a CE foi obtida aumentando-se os nutrientes proporcionalmente, o que, possivelmente, originou uma interação entre os componentes iônicos da solução nutritiva, prejudicando a absorção de água e/ou algum nutriente e levando as plantas ao desequilíbrio nutricional nas maiores concentrações testadas. Outras possíveis variáveis que possam ter influenciado a produção de estolhos são as distintas condições ambientais entre os anos de realização desses trabalhos.

Em trabalho realizado por Bish et al. (2001) com mudas matrizes da cultivar Oso Grande em 1995 e 1996, verificou-se uma maior produção de estolhos no ano de 1996 , sendo o aumento de 59\% atribuído à redução da concentração da solução nutritiva de $1,72 \mathrm{dS} / \mathrm{m}$ para 0,7 $\mathrm{dS} / \mathrm{m}$ (redução de $1,0 \mathrm{dS} / \mathrm{m}$ ), proporcionando uma produção de 84 estolhos por planta matriz. No presente trabalho observou-se uma produção de 73,5 estolhos por planta matriz com redução de $38,7 \%$ decorrente do aumento de $1,2 \mathrm{dS} / \mathrm{m}$ para $2,0 \mathrm{dS} / \mathrm{m}$. A redução da concentração da solução nutritiva empregada por Bish et al. (2001) no ano de 1996 foi em decorrência da elevada incidência de queima de bordos das folhas observada no ano anterior, o que, também, foi observado visualmente no presente trabalho em concentrações 


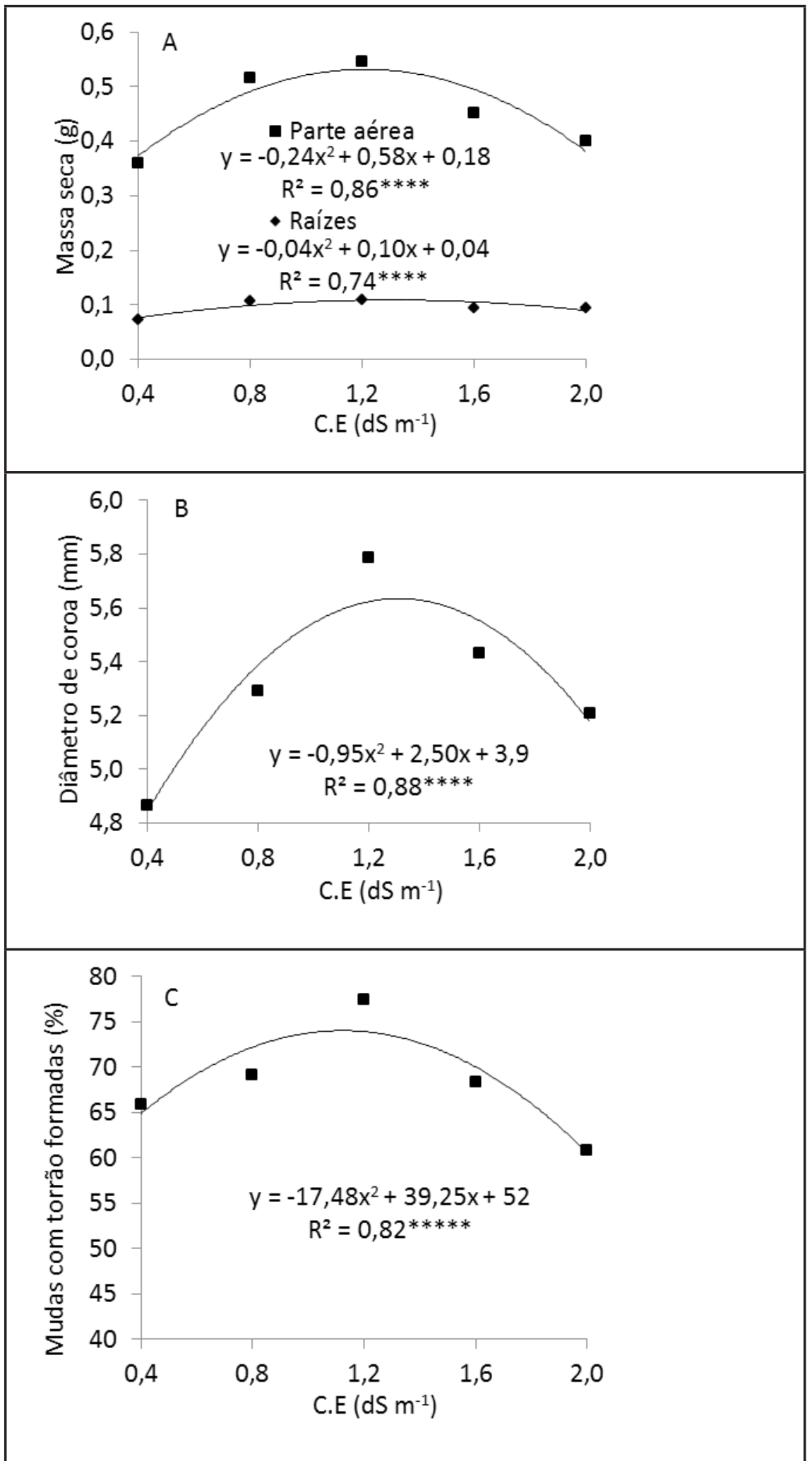

Figura 2. Massa seca da parte aérea e raízes (A); diâmetro de coroa (B) e porcentagem de formação de mudas com torrão $(\mathrm{C})$, produzidas a partir de estolhos, coletados de plantas matrizes de morangueiro das cultivares Oso Grande e Camino Real cultivadas sob cinco concentrações da solução nutritiva $(0,4 ; 0,8 ; 1,2 ; 1,6$ e $2,0 \mathrm{dS} / \mathrm{m})$. **** e ***** $=\mathrm{p} \leq 0,15 \mathrm{e}$ $\mathrm{p} \leq 0,20$, respectivamente shoot and root dry mass (A), crown diameter (B) and percentage of plants with plugs $(\mathrm{C})$ produced from Oso Grande and Camino Real runner tips collected from stock plants grown under five concentrations of the nutrient solution $(0.4 ; 0.8 ; 1.2 ; 1.6$; and $\left.2.0 \mathrm{dS} \mathrm{m}^{-1}\right) . * * * *$ and $* * * * *=\mathrm{p} \leq 0.15$ and $\mathrm{p} \leq 0.20$, respectively $\}$. Santa Maria, UFSM, 2012. superiores a $1,2 \mathrm{dS} / \mathrm{m}$.

$\mathrm{O}$ aumento de estolhos necrosados em função da elevação da CE (Figura $1 \mathrm{~B}$ ) foi uma possível resposta às deficiências no fornecimento de água imposta ao estolho pela planta matriz. Os estolhos são os principais drenos das plantas matrizes, portanto demandam grandes quantidades de água e fotoassimilados. Em caso de restrição de água, os estolhos são rapidamente afetados na tentativa da planta matriz de minimizar perdas e sobreviver (Blancke \& Coocke, 2004). A restrição de água e/ou alguma deficiência nutricional pode $(\mathrm{m})$ ser prejudicial(is) ao crescimento e desenvolvimento de novos órgãos e regiões meristemáticas na cultura do morangueiro (Palencia et al., 2010), o que pode explicar, além da necrose dos estolhos, a menor taxa de formação de mudas com torrão por meio de estolhos oriundos de plantas matrizes cultivadas sob concentrações diferentes de 1,1 $\mathrm{dS} / \mathrm{m}$ (Figura 1B e 1C).

$\mathrm{O}$ diâmetro de coroa e a massa seca dos estolhos não foram afetados pelas concentrações da solução nutritiva, assim como verificado por Janisch et al. (2012). O diâmetro de coroa diferiu das médias encontradas por Giménez et al. (2008) e Oliveira et al. (2010), podendo tais diferenças serem atribuídas ao manejo e sistema de cultivo empregado na produção de estolhos. No presente trabalho, a coleta dos estolhos ocorreu semanalmente, o que, segundo Durner et al. (2002), proporciona estolhos de maior diâmetro de coroa e uniformidade de mudas formadas. De acordo com os critérios de Cocco et al. (2010), os estolhos produzidos, assim como as mudas com torrão formadas, apresentaram diâmetro de coroa satisfatório para proporcionar uma produção precoce de frutos a campo sem diferenças na produção total de frutos.

Plantas matrizes de morangueiro de ambas as cultivares observadas apresentaram seu crescimento fortemente reduzido com o aumento da concentração da solução nutritiva. Essa redução mostrou que o morangueiro é uma espécie sensível à utilização de concentrações superiores a $0,4 \mathrm{dS} / \mathrm{m}$, diferindo da classificação de Martinez Barroso \& Alvarez (1997), que consta- 


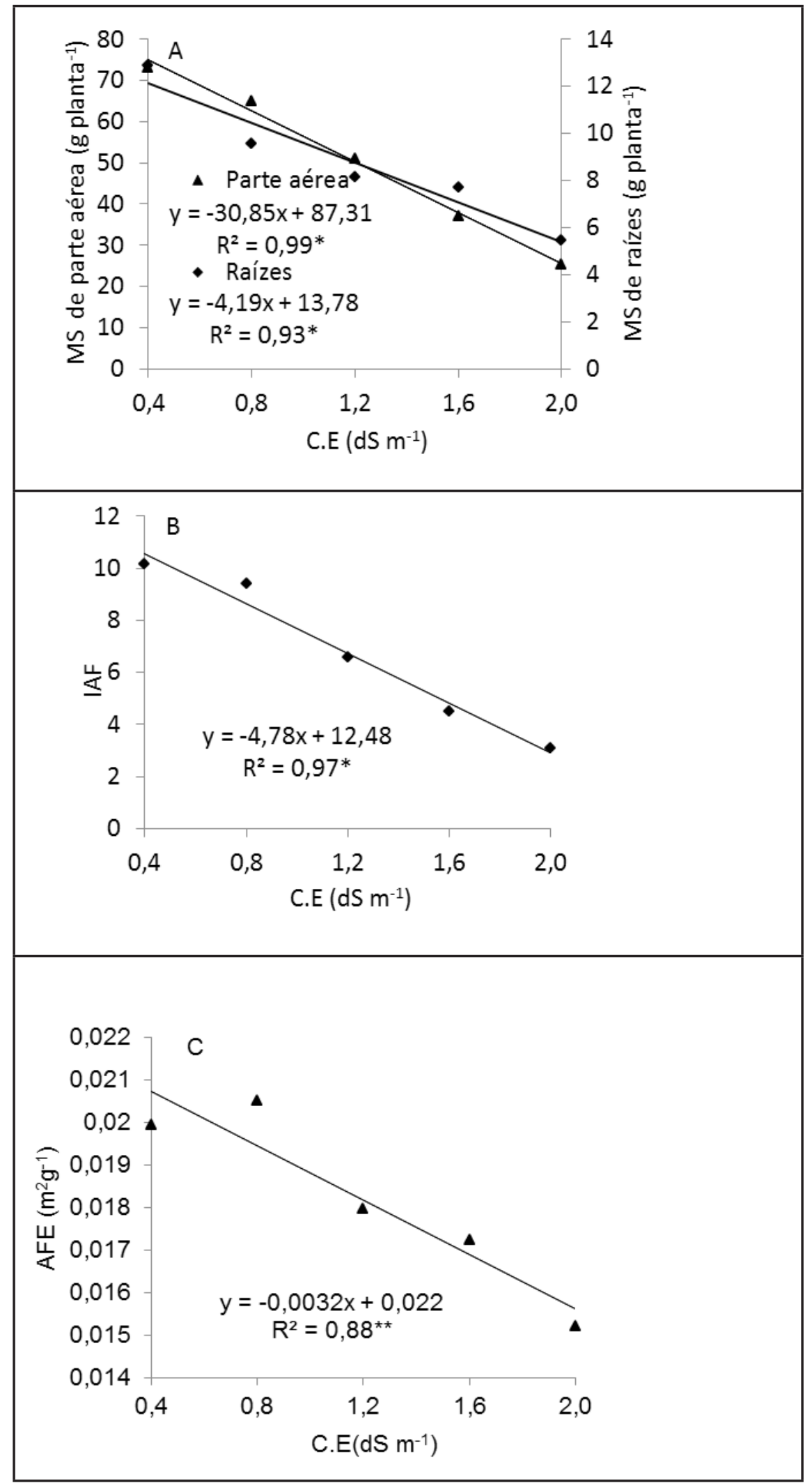

Figura 3. Massa seca de parte aérea e raízes (A); índice de área foliar (IAF) (B) e área foliar específica (AFE) (C) de plantas matrizes de morangueiro das cultivares Oso Grande e Camino Real cultivadas sob cinco concentrações da solução nutritiva $(0,4 ; 0,8 ; 1,2 ; 1,6$ e $2,0 \mathrm{dS} / \mathrm{m}) .{ }^{*} \mathrm{e}^{* *}=\mathrm{p} \leq 0,01, \mathrm{p} \leq 0,05$, respectivamente shoot and root dry mass (A); leaf area index (IAF) (B) and specific leaf area (C) of Oso Grande and Camino Real stock plants grown under five concentrations of the nutrient solution $(0.4 ; 0.8 ; 1.2 ; 1, .6$; and $2.0 \mathrm{dS} / \mathrm{m})$. $*$ and $* *=p \leq 0.01, p \leq 0.05$, respectively $\}$. Santa Maria, UFSM, 2012. taram a sensibilidade do morangueiro a concentrações superiores a $1,4 \mathrm{dS} / \mathrm{m}$. No presente trabalho observou-se redução de $65,8 \%$ da parte aérea em plantas cultivadas sob a $\mathrm{CE}$ de $2,0 \mathrm{dS} / \mathrm{m}$ em comparação a $0,4 \mathrm{dS} / \mathrm{m}$, valor próximo ao observado por Rahimi et al. (2011), o qual observou redução de $62 \%$ no crescimento de plantas de morangueiro devido aos efeitos da salinidade.

$\mathrm{O}$ crescimento das plantas matrizes $\mathrm{e}$ o índice de área foliar (Figuras 3A e 3B) foram afetados pela redução no número de folhas, ocasionada pela elevação da $\mathrm{CE}$, o qual apresenta uma resposta polinomial $\left(y=-12,22 x^{2}+13,92 x+47,27\right.$; $\mathrm{R}^{2}=0,93$ ) com uma redução próxima a $50 \%$ em relação a $\mathrm{CE}$ de $0,4 \mathrm{dS} / \mathrm{m}$ e resulta em média de 26 folhas em plantas matrizes cultivadas na CE 2,0 $\mathrm{dS} / \mathrm{m}$. Essas reduções estão relacionadas à concentração de sais dos fertilizantes usados no preparo das soluções nutritivas, resultando na redução da disponibilidade de água e, consequentemente, estresse hídrico, a partir do qual surge a deficiência de cálcio e, com ela, a necrose de folhas jovens recém emitidas, impossibilitando seu desenvolvimento e crescimento (Lieten, 2000; Sonsteby et al., 2009; Palencia et al., 2010). Reduções no crescimento de plantas de morangueiro com aumentos da concentração da solução nutritiva foram verificados por Portela et al. (2012) e Andriolo et al. (2009).

$\mathrm{O}$ uso de soluções nutritivas concentradas é uma prática que permite reduzir o crescimento vegetativo quando for desejado. Li \& Stanguellini (2001) e Romero-Aranda et al. (2001) observaram que essa prática foi possível na cultura do tomateiro sem prejudicar a produção de frutos. No cultivo de plantas matrizes de morangueiro, essa prática deve ser evitada, pois reduz o crescimento das folhas e a emissão de estolhos e aumenta o número de estolhos necrosados, reduzindo o número de estolhos viáveis para a produção de mudas com torrão.

As plantas matrizes cultivadas na CE $0,4 \mathrm{dS} / \mathrm{m}$ apresentaram maior crescimento da parte aérea e raízes, porém não apresentaram maior produção de estolhos. O maior crescimento do sistema radicular de plantas expostas a 
baixas concentrações de nutrientes nas soluções nutritivas se justifica pela necessidade da busca de nutrientes (Lambers et al., 2008), o que é possível sob condições atmosféricas de alta demanda evaporativa (Sonneveld \& Voogt, 2009). Janisch et al. (2012) avaliaram doses de nitrogênio no cultivo de plantas matrizes de morangueiro e verificaram que a menor dose não foi limitante ao crescimento das plantas, que apresentaram maior crescimento radicular, confirmando o resultado do atual trabalho.

A área foliar específica (AFE), indicador do acúmulo de massa foliar, foi reduzida com o aumento da concentração de sais na solução nutritiva (Figura 3C). Segundo Lambers et al. (2008), a redução da AFE é desencadeada pelo estresse de água, de forma a limitar a expansão foliar, criando barreiras para a perda de água via transpiração. Isso é uma estratégia para melhorar a eficiência no uso da água (Rahimi et al., 2011).

A formulação e o manejo da solução nutritiva de forma adequada são fundamentais para evitar redução do potencial de produção de estolhos, conforme o sistema de cultivo empregado. Em sistemas de cultivo fechado com uso de substratos, poderá ser usada uma solução nutritiva diluída e com um coeficiente de drenagem mais elevado para evitar o acúmulo de sais no substrato. Em sistemas de cultivo aberto, em que o fornecimento de nutrientes é realizado de forma descontínua e alternado com irrigação usando somente água, é importante que se faça o emprego de soluções mais concentradas, evitando o surgimento de deficiências nutricionais nas plantas.

O emprego de sistemas de cultivo sem solo com reaproveitamento da solução nutritiva, aliado ao uso de soluções nutritivas com concentrações iônicas reduzidas e equilibradas na produção de estolhos, reduz gastos com fertilizantes, minimiza a contaminação do solo e da água e possibilita uma produção de estolhos satisfatória, tanto em quantidade como em qualidade. O número de estolhos viáveis produzidos, aliado à percentagem de mudas formadas a partir dessas, são fundamentais na produção comercial de mudas de morangueiro em torrão, afetando diretamente os custos e a rentabilidade do sistema de produção.

No presente trabalho verificou-se uma maior produção de estolhos, formação e qualidade de mudas em concentrações compreendidas entre $0,8 \mathrm{e}$ $1,1 \mathrm{dS} / \mathrm{m}$, o que diferiu da concentração de 1,6 dS/m, recomendada nos Estados Unidos por Paranjpe et al. (2003) e é similar à recomendação de Bish et al. (2001), na qual a maior produção de estolhos foi verificada na CE de 0,7 $\mathrm{dS} / \mathrm{m}$.O crescimento das plantas matrizes de morangueiro foi reduzido com o aumento da concentração da solução nutritiva na faixa de CE entre 0,4 e $2,0 \mathrm{dS} / \mathrm{m}$. A produção de estolhos e de mudas com torrão foram maiores com condutividade elétrica na faixa entre 0,8 a $1,1 \mathrm{dS} / \mathrm{m}$ na fertirrigação das plantas matrizes em sistema fechado de cultivo em areia.

\section{AGRADECIMENTOS}

Ao Conselho Nacional de Desenvolvimento Científico e Tecnológico (CNPq), pelo auxílio financeiro e pela concessão da bolsa de mestrado para Odair José Schmitt.

\section{REFERÊNCIAS}

ANDRIOLO, JL; JANISCH, DI; DAL PICIO, M; SCHMITT, OJ; LERNER, MA. 2014. Nitrogen accumulation and monitoring by strawberry stock plants for runner tips production. Horticultura Brasileira 32: 273-279.

ANDRIOLO, JL; JANISCH, DI; SCHMITT, OJ; VAZ, MAB; CARDOSO, FL; ERPEN, L. 2009. Concentração da solução nutritiva no crescimento da planta, na produtividade e na qualidade de frutas do morangueiro. Ciência Rural 39: 684-690.

ARMEFLHOR. 2006. Rapport technique sur la production de plants Fraisimotte ${ }^{\circledR}$ a l'ile de la reunion. Saint-Pierre: Association Réunionnaise pour la modernization de l'Economie fruitière légumière et hortícola. 69p. Disponível em: www.armeflhor.fr. Acessado em 12 de janeiro de 2013.

BISH, EB; CANTLIFFE, DJ; CHANDLER, CK. 2001. A system for producing large quantities of greenhouse grown strawberry plantlets for plug production. HortTechnology 11: 636-638.

BLANCKE, MM; COOCKE, DT. 2004. Effect of flooding and drought on stomatal activity, transpiration, photosynthesis, water potential and water channel activity in strawberry stolons and leaves. Plant Growth Regulation
42: $153-160$

COCCO, C; ANDRIOLO, JL; ERPEN, L; CARDOSO, FL; CASAGRANDE, GS. 2010. Desenvolvimento e produtividade do morangueiro influenciados pelo diâmetro da coroa e período de crescimento de mudas. Pesquisa Agropecuária Brasileira 45: 730736.

DURNER, EF; POLING, EB; MAAS, JL. 2002. Recent advances in strawberry plug transplant technology. HortTechnology 12: 545-550.

FARINA, E; ALERA, C; PAERNIANI, T; PALAGI, M. 2003. Mulching as a technique to reduce salt accumulation in soilless culture. Acta Horticulturae 609: 459-466.

FERREIRA, DF. 2003. Sisvar versão 4.2. DEX/ UFLA.

GIMÉNEZ, G; ANDRIOLO, JL; JANISCH, DI; GODOI, RS. 2008. Closed soilless growing system for producing strawberry bare root transplants and runner tips. Pesquisa Agropecuária Brasileira 43: 1757-1761.

GODOI, RS; ANDRIOLO, JL; GIMÉNEZ, G; JANISCH, DJ; CARDOSO, FL; VAZ, MAB. 2009. Produção e qualidade do morangueiro em sistemas fechados de cultivo sem solo com emprego de substratos. Ciência Rural 39: 1039-1044.

HENNION, B; VESCHAMBRE, D. 1997. La fraise: maîtrise de la production. Paris: CTIFL. 299p.

JANISCH, DI; ANDRIOLO, JL; TOSO, V; SANTOS, KGF; SOUZA, JM. 2012. Nitrogen for growth of stock plants and production of strawberry runner tips. Bragantia 71: 394-399.

LAMBERS, H; CHAPIN, FS; PONS, TL. 2008. Plant physiological ecology. Berlin: Springer Verlag.

LI, YL; STANGHELLINI, C. 2001. Analysis of the effect of EC and potencial transpiration on vegetative growth of tomato. Scientia Horticulturae 89: 9-21.

LIETEN, F. 2000. Recent advances in strawberry plug transplant technology. Acta Horticulturae 513: 383-388.

MARTINEZ-BARROSO, C; ALVAREZ, CE. 1997. Toxicity symptoms and tolerance of strawberry to salinity in the irrigation water. Scientia Horticulturae 71: 177-188.

OLIVEIRA, CS; COCCO, C; ANDRIOLO, JL; BISOGNIN, DA; ERPEN, L; FRANQUEZ, G. 2010. Produção e qualidade de propágulos de morangueiro em diferentes concentrações de nitrogênio no cultivo sem solo. Revista Ceres 57: 554-559.

PALENCIA, P; MARTINEZ, F; RIBEIRO, E; PESTANA, M; GAMA, F; SAAVEDRA, T; VARANNES, A; CORREIA, PJ. 2010. Relationship between tipburn and leaf mineral composition in strawberry. Scientia Horticulturae 126: 242-246.

PARANJPE, AV; CANTLIFFE, DJ; LAMB, EM; STOFFELLA, PJ; POWELL, C. 2003. Winter strawberry production in greenhouses using soilless substrates: an alternative to methyl bromide soil fumigation. Proceedings of the Florida State for Horticultural Society 116: 98-105.

PORTELA, IP; PEIL, RMN; ROMBALDI, CV. 
2012. Efeito da concentração de nutrientes no crescimento, produtividade e qualidade de morangos em hidroponia. Horticultura Brasileira 30: 266-273.

R A H IMI, A; B I GL ARIFARD, A ; MIRDEHGHAN, H; BORGHEI, SF. 2011. Influence of $\mathrm{NaCl}$ salinity on growth analysis of strawberry cv. Camarosa. Journal of Stress Physiology \& Biochemistry 7: 145-156.

REISSER JÚNIOR, C; TAVARES, EVQ; TIMM, LC; ESTRELA, CC; ANTUNES, LEC; CUNHA, NG. 2009. Solo e manejo da água. In: TIMM, LC; TAVARES, EVQ; REISSER JUNIOR, C; ESTRELA, CC. (eds). Morangueiro irrigado: aspectos técnicos e ambientais do cultivo. Pelotas: UFPEL. p.51-91.
ROMERO-ARANDA，R；SORIA，T; CUARTERO, J. 2001. Tomato plant water uptake and plant water relationships under saline growth conditions. Plant Science 160: 265-272.

SCHMITT, OJ; ANDRIOLO, JL; TOSO, V; JANISCH, DI; PICIO, MD; LERNER, MA. 2012. Frigoconservação das pontas de estolões na produção de muda com torrão e frutas de morangueiro. Ciência Rural 42: 955-961.

SHAW, VD; LARSON, K. 2002. Strawberry plant named Camino Real. United States Plant Patent, PP13079, 15 Oct. 2002. Disponível em: http://www.patentgenius.com/patent/ PP13079.html. Acessado em 20 de agosto de 2011.

SONNEVELD, C; VOOGT, W. 2009. Plant nutrition of greenhouse Crops. New York: Springer. 431p.

SONSTEBY, A; OPSTAD, N; MYRHEIM, U; HEIDE, O. 2009. Interaction of short day and timing of nitrogen fertilization on growth and flowering of 'Korona' strawberry (Fragaria $x$ ananassa Duch.). Scientia Horticulturae 123: 204-209.

SPSS Incorporation for Windows. 2004. Statistical package for the social sciences. Release 13.0, Chicago, Illions: SPSS Inc.

VOTH, V.; BRINGHURST, RS. 1989. Strawberry plant called 'OsoGrande'. United States Plant Patent. PP6578, 31 Jan. 1989. Disponível em: http://www.patentgenius.com/patent/PP6578. html. Acessado em 20 de agosto de 2011. 